\title{
El dispositivo de la Modernidad política en R. Esposito y G. Duso. Elementos para un diálogo entre ontología de la actualidad e historia conceptual ${ }^{*}$
}

\author{
The Dispositif of Political Modernity in R. Esposito and \\ G. Duso. Elements for a dialogue between Ontology of the \\ Present and Conceptual History
}

\begin{abstract}
Resumen: El presente trabajo plantea un diálogo entre la ontología de la actualidad en clave biopolítica defendida por Roberto Esposito y la historia conceptual como filosofía política de Giuseppe Duso. Además de presentar en sus líneas básicas ambas propuestas, el objetivo es mostrar, más allá de las diferencias, las convergencias en su deconstrucción de los conceptos políticos modernos, por un lado, y en las tareas que asignan a la filosofía política, por el otro.

Palabras clave: Historia Conceptual, Biopolítica, Esposito, Duso, Modernidad política, filosofía política
\end{abstract}

HÉCTOR VIZCAÍNO REBERTOS ${ }^{* *}$

\begin{abstract}
This paper presents a fruitful dialogue between the conceptual history as a political philosophy of Giuseppe Duso and the ontology of present in biopolitics key defended by Roberto Esposito. Besides presenting the basic lines both proposals, the aim of this paper is to show, beyond its differences, the convergences in their deconstruction of modern political concepts, on the one hand, and in the tasks assigned to political philosophy, on the other.
\end{abstract}

Keywords: Conceptual History, Biopolitics, R. Esposito, G. Duso, Political Modernity, Political Philosophy

Fecha de recepción: 06/06/2016. Fecha de aceptación: 06/09/2016.

* Este trabajo se beneficia de una beca de carácter pre-doctoral del Subprograma «Atracció de Talent» de la Universtat de València y se enmarca en el grupo de investigación «Historia Conceptual y crítica de la Modernidad» (GIUV2013-037) de la Universtat de València. Si no se hace referencia explícita a una versión española de las obras o artículos citados, la traducción es del autor.

** Personal investigador en Formación en el Departamento de Filosofía de la Universitat de València gracias a una beca pre-doctoral. Mis líneas de investigación se orientan hacia un diagnóstico de la génesis, lógica y crisis de la modernidad política desde una perspectiva histórico-conceptual, prestando especial atención al pensamiento filosófico-político italiano actual. Algunas de mis últimas publicaciones: F. Oncina, N. Miravet y H. Vizcaíno (eds.), Conceptos nómada: Auto-determinación, Valencia, PUV, 2014; edición del número monográfico «Cambio de paradigma en la filosofía política contemporánea: la Italian Theory», Pasajes. Revista de pensamiento contemporáneo, $\mathrm{n}^{\circ}$ 49,2016. Contacto: hector.vizcaino@uv.es. 


\section{Introducción}

A mediados de marzo de 2016, la editorial Einaudi publicó la última obra de Roberto Esposito, Da fuori. Una filosofia per l'Europa, en la que, en parte, retoma y desarrolla la genealogía del denominado Italian Thought ya planteada en trabajos anteriores (cf. Esposito, 2015 y 2016b). Esposito defiende la tradición de la filosofía política italiana como un horizonte de pensamiento, muy vinculado a las temáticas de la política, de la historia y de la vida, que precede y desborda el plexo de lógicas, léxicos e instituciones que conforman la Modernidad, capaz, por ello, de ofrecernos herramientas para comprender e intervenir en el contexto de la actual globalización post-estatal.

Llama la atención, sin embargo, que en su reconstrucción del pensamiento italiano más actual, en el que destaca los trabajos de Negri, Agamben, Cacciari, Marramao y el suyo propio, Esposito no incluya la historia conceptual -salvo una brevísima referencia, en su última obra, a la recepción italiana de Schmitt (2016: 186). Y sorprende no sólo porque -a través de esa operación de déterritorialisation que, según Esposito, caracteriza el Italian Thought- una de las recepciones críticas más fructíferas de la Begriffsgeschichte se haya dado en Italia, especialmente la que cristaliza en la propuesta de historia conceptual como filosofía política de Giuseppe Duso y el Gruppo di ricerca sui concetti politici de la Università di Padova; sino porque, además, uno de sus objetivos fundamentales es, precisamente y en consonancia con las coordenadas trazadas por Esposito, el de deconstruir el dispositivo conceptual moderno y pensar más allá del mismo la nueva realidad política europea, proponiendo para ello la categoría de federalismo ${ }^{1}$. En parte, la ausencia de referencias a la historia conceptual se debe a que Esposito (2006) en un virulento editorial de Filosofia Politica trató de romper cualquier tipo de vínculo entre aquélla y la biopolítica, como ontología de la actualidad, que defiende desde Immunitas.

A partir del dato de la cada vez más inextricable e inmediata implicación que en la modernidad se establece entre la política y la vida humana, en su dimensión específicamente biológica, el editorial comienza reivindicando la «biopolítica como "la situación concreta" en la que desde hace tiempo nos movemos y el horizonte filosófico destinado a interpretarla» (Esposito, 2006, 5). Esta reivindicación pasa por una doble toma de distancias. Primero con las otras versiones de la biopolítica, especialmente con la del Agamben del primer volumen de Homo Sacer, que, a juicio de Esposito, eterniza, remontando a la Antigüedad, una dimensión de lo político, como es la del poder soberano que, en su opinión, es moderna y sólo moderna. La segunda, y a partir de la reivindicación de la modernidad de la biopolítica, carga contra el planteamiento histórico-conceptual de Giuseppe Duso. Lo hace, con un juicio poco ponderado sobre el que se volverá, reduciéndolo a «una refinada metodología académica» que no puede integrar en su interior «una modalidad radical de pensar activamente nuestra contemporaneidad, como quiere ser, en sus diferentes acepciones, la biopolítica con la que no tiene afinidades de intenciones o de inspiraciones» (Ibid, 8). En opinión de Esposito, son dos perspectivas intelectuales incompatibles, en primer lugar, por lo que respecta al método empleado en la investigación y, sobre todo, en su concepción de la modernidad.

1 Para un exposición sintética, cf. Chignola-Duso (2009, 115-157 y 357-375), Duso (1998) y Sánchez Mandingorra (2016). 
Cabe preguntase si, como pretende Esposito, los horizontes filosóficos de la historia conceptual y de la ontología de la actualidad realmente están tan alejados. El objetivo de este trabajo es entablar un diálogo entre estas dos corrientes de la filosofía política que vienen de Italia, mostrando sus coincidencias en el diagnóstico que realizan del presente a partir de una muy peculiar genealogía compartida de la Modernidad como dispositivo del orden y del carácter que en ella asume el concepto, que les conduce al pronóstico de la necesidad de pensar Europa más allá de la soberanía. La tesis que se defiende es que ambos horizontes no sólo convergen en su diagnóstico-pronóstico, es decir, «en afinidades de intenciones», sino que en la deconstrucción del dispositivo de la Modernidad que opera la biopolítica, precisamente el elemento donde Esposito afirma que se produce la diferencia más irreconciliable entre ambas corrientes, subyace un planteamiento histórico-conceptual.

\section{La modernidad política como dispositivo del orden}

Para entablar este diálogo a propósito de la Modernidad política, hay que comenzar señalando que ambas perspectivas parten de un diagnóstico compartido sobre la génesis y la crisis de los conceptos políticos modernos, un diagnóstico que -fraguado como una koiné en la Italia de los años 80, especialmente, en torno a la revista Il Centauro ${ }^{2}$ - sigue operando en sus trabajos más recientes. Dicho dictamen pone de manifiesto que los conceptos políticos modernos (individuo, derechos, libertad, igualdad, soberanía, representación, Estado, etc.) no nos permiten comprender las dinámicas políticas actuales de la globalización post-estatal, pues, a través de la lección schmittiana, estos autores asumen que lo político excede la dimensión de la estatalidad del ius publicum europaeum. De ahí que lo que ya no funciona no sean tanto los conceptos singulares mencionados sino, más bien, el mismo marco conceptual e institucional en el que se inscriben. Partiendo del presente, de la necesidad de comprender e intervenir en él, ambos autores movilizan una interrogación filosófica con la que evidenciar la génesis, la lógica y las aporías de esos conceptos con los que, habitualmente, pensamos la política y cuyo poder de significación, sin embargo, parece desfallecer en el momento en el que el horizonte de sentido que los hacía significativos ha entrado definitivamente en crisis. De ahí, el proyecto compartido de pensar lo político más allá del Estado, del nexo soberanía-representación, es decir, de las categorías de la modernidad política, para comprender en su especificidad y singularidad las dinámicas políticas actuales. Dicho esto, pasemos al análisis concreto que ambas perspectivas ofrecen de la génesis de la modernidad como dispositivo del orden.

La historia conceptual que propone Duso no puede entenderse, como despectivamente la caracteriza Esposito, como una «refinada metodología académica» con la que trabajar a priori sobre los conceptos. No puede reducirse a un método porque parte, precisamente, de la puesta en cuestión del vínculo que en la modernidad se establece entre teoría y praxis, es decir, de la idea de método como «un modelo que pretende basarse no tanto en la experiencia, como en sí mismo, en su propio rigor [con el que] organizar de manera racional la sociedad y el mando que en ésta se ejerce» (Duso, 2016, 35). Entre los resultados más

2 Para la constelación intelectual italiana que orbita en torno a Il Centauro (1981-1986) y el diagnóstico epocal sobre la crisis de lo político que en ella se elabora, cf. Esposito (2008, 15 y ss.) y Gentili (2008, 9-48; 2011, 109-165). 
originales de este análisis destaca la caracterización que se hace del concepto de «concepto» y la función que en la modernidad asume. El objeto preeminente de la historia conceptual son los conceptos políticos modernos porque, propiamente hablando, dice Duso, sólo hay conceptos modernos, en un sentido muy preciso: para que se pueda dar una construcción teórica pura es necesario la existencia de unas unidades ideales caracterizadas, a la vez, por la operatividad y abstracción sobre la experiencia concreta que permita, a priori, proporcionar una respuesta científica formal, un modelo de construcción del orden (político). Por tanto, los conceptos, caracterizados de este modo, son modernos y sólo modernos. Antes que un método así entendido, la historia conceptual trabaja «metódicamente en la problematización de cuanto, en general, es dado por autoevidente» (Chignola-Duso, 2009, 33). Y lo que se presenta como evidente son las herramientas, esto es, los conceptos con los que comprendemos tanto el presente como el pasado y, en consecuencia, la política. En ellos opera a menudo un presupuesto incuestionado por el que se cree que los conceptos, entendidos como entidades intelectuales eternas, designan realidades y valores objetivos y universales. Sin embargo, si se piensa con cierto detenimiento en ello, rápidamente puede constatarse que lejos de ser universales y unívocos, los conceptos políticos mencionados, así como la noción misma de concepto que está a su base, tienen un origen muy concreto que pone de manifiesto su contingencia, contingencia en la que, por otra parte, habita la posibilidad de pensar la política de otra manera. Por ello, Duso propone interrogar los conceptos modernos metódica y filosóficamente, es decir, pedir razón de su presunta universalidad y objetividad, preguntándose «¿de dónde vienen, qué presupuestos tienen, cuál es su lógica y qué aporías se revelan en ellos?» (Duso, 1998, 36). En estas preguntas se entrelazan una perspectiva genealógica («de dónde vienen») y otra deconstructiva («cuál es su lógica y qué aporías se revelan en ellos») con las que evidenciar que: «en el ámbito del denominado iusnaturalismo moderno nació un verdadero dispositivo para pensar la política, que se plantea como radical negación de una larga tradición de filosofía política, y que condiciona el modo de entender la política hoy. Se trata de un mecanismo que considera el orden de la sociedad como producto del sujeto, la política como pensable mediante la forma jurídica, el poder necesario en la sociedad como impersonal y fundado sobre la voluntad de los individuos y sus derechos, igualdad y libertad in primis. En este mecanismo, los conceptos tienen una función lógica propia, tanto más precisa cuanto menos ligada a las realidades concretamente determinadas» (Chignola-Duso, 2009, 355).

La historia conceptual, por tanto, no sólo no se identifica sino que se opone a la historia de las palabras, en el momento en que se asume como principio la distinción entre concepto y palabra heredada de la Begriffsgeschichte, ya que evita tratar los conceptos aisladamente para centrar el análisis en el principio organizativo, el dispositivo, que los vincula e integra lógicamente. Desde esta perspectiva, los conceptos políticos con los que aún hoy pensamos la política surgen de una ruptura radical (Trennung) que tiene lugar en el siglo XVII, con las doctrinas del contrato social ${ }^{3}$, concretamente en Hobbes, y que cristalizan en las instituciones políticas surgidas entre los siglos XVIII y XIX. La ruptura a la que apela no supone una mera transformación del significado de las palabras, sino, más bien, la negación de todo el

3 Para la ruptura que operan las teorías del contrato social y la neutralización de la pregunta por la justicia y el buen gobierno sustituida por la de la legitimidad del poder (soberanía), cf. Duso (1987, 7-49; 1999, 10-21 y 91-97). 
horizonte de sentido anterior y el nacimiento de una manera nueva de entender el hombre y la política. Asumiendo la distinción entre palabra y concepto mencionada, Duso sostiene que lo que tiene lugar en el siglo XVII no es un cambio en el significado de términos como «política», «sociedad», «representación», o «poder», sino una transformación del concepto que estas palabras vehiculan y expresan, pues, «cuando se atribuyen cambios históricos a los conceptos, nos encontramos en realidad frente al nacimiento de nuevos conceptos que usan para comunicarse viejas palabras, ya conocidas» (Duso, 1998, 39).

A este enfoque genealógico, se añade otro deconstructivo que, a la vez, desmonta los conceptos, examina con detalle su funcionamiento constelativo y pone de manifiesto las aporías que integran, de forma constitutiva, no sólo los conceptos singulares y su disposición lógica, sino su cristalización institucional, especialmente, en el nexo que vincula representación y soberanía en la constitución de la democracia representativa. Con Hobbes se inaugura un «un verdadero dispositivo teórico de construcción y legitimación del poder» (Duso, 2009, 335) con el que, a través de la racionalidad formal y la cientifización de la ética que se impone con la nueva ciencia del derecho natural, se neutraliza el principio organizador del horizonte de pensamiento antiguo y premoderno, esto es, la idea de justicia pierde el papel central que asumía en el antiguo principio del buen gobierno. Se neutraliza la pregunta por la justica ya que, no tanto la pregunta como las respuestas dogmáticas que se dan son una fuente permanente de desorden y conflicto (piénsese en las guerras de religión). La pregunta por la justicia es reemplazada por una construcción racional cuyo punto de partida es el individuo, los derechos del individuo (especialmente, la igualdad y la libertad) a partir de los que se constituye un cuerpo político unitario dentro del cual se ejerce legítimamente la coacción para salvaguardar la paz y los derechos individuales. La operación hobbesiana reclama un discurso científico con valor universal y vinculante que, inspirado en el rigor geométrico, elabore una concepción unívoca de lo justo con la que alcanzar no ya el summum bonum, sino evitar la muerte violenta, y garantizar así el fin propio de la vida de los hombres: la autoconservación. La solución hobbesiana concluye que lo justo es obedecer las leyes (primera y segunda ley de la naturaleza), reduciendo el problema de la justicia a una respuesta formal con la que si, de un lado, se neutraliza el conflicto que entrañan las diferentes concepciones de la justifica, al mismo tiempo identifica lo político a lo jurídico.

El proceso lógico inaugurado por Hobbes que está a la base de este dispositivo de legitimación del poder -que, aunque agotado, según Duso aún perdura en nuestra forma de entender la política- parte de la tajante separación entre moral y política, puesto que aquélla no es suficiente para vivir en sociedad, por lo que es necesario un poder que imponga leyes racionales y legítimas. Dicho poder (soberanía) tiene que estar basado en la igualdad natural de los individuos y fundado racionalmente. Por tanto, ahí se afirma el principio de igualdad, que rompe con los lazos de sumisión personal entre gobernante y gobernado propios de la doctrinas del buen gobierno. La ley que se instaura para garantizar la seguridad y supervivencia de los individuos iguales es producto de un proceso de autorización libre. Los individuos son el presupuesto, el fundamento de la sociedad, al constituirla mediante la manifestación libre de la voluntad que da lugar al contrato social, tras el cual quedan sometidos al soberano. Esta sumisión al sujeto colectivo no es considerada servidumbre, sino, al contrario, el único modo de ser libres. 
Si éste es el dispositivo de legitimación del poder de la modernidad, una de sus grandes aporías, señala Duso, es la despolitización y reducción de la pluralidad de los sujetos a la «unidad política» que instaura el concepto de soberanía, el perno que sintetiza y articula todos los engranajes que hacen funcionar este mecanismo conceptual, rompiendo con la relación personal de sumisión que implica la lógica del gobierno. Frente a este horizonte, partiendo de la igualdad y la libertad del individuo, el poder designa una dimensión impersonal del sometimiento político, sometimiento u obediencia que no es a alguien particular, sino al cuerpo político representado y, en el fondo, a uno mismo, en la medida en que quien obedece acepta la orden como regla del propio comportamiento sobre la base de su propia voluntad (cf. Duso, 2009, 215 y ss.) La aporía de este dispositivo es la férrea unidad política que constituye el nexo soberanía-representación, planteando un problema que no puede resolverse desde esta lógica: la neutralización de la alteridad y particularidad de los individuos que conforman el pueblo.

Por lo que respecta a Esposito, podemos encontrar afinidades con el análisis descrito en la genealogía de la modernidad política que elabora en Bíos. Esta genealogía es la que moviliza el paradigma inmunitario que el autor napolitano propone como herramienta heurística con la que describir la lógica que inaugura la modernidad en la que se anuda, con un vínculo simultáneo y antinómico de afirmación y negación, vida y política. A partir del doble significado, médico/biológico y jurídico, del término «inmunidad» opuesto al de «comunidad» en su sentido latino explorado en Communitas en el que ahora no podemos detenernos, afirma que «la inmunidad no es únicamente la relación que vincula la vida con el poder, sino el poder de conservación de la vida. [...] De acuerdo con esta perspectiva, la política no es sino la posibilidad, o el instrumento, para mantener con vida la vida» (Esposito, 2011, 74). Que «desde siempre» ha habido un vínculo entre vida y política es obvio, pero no lo es tanto que «sólo a partir de un determinado momento, precisamente en coincidencia con el origen de la modernidad, esa necesidad de autoaseguramiento haya sido reconocida ya no simplemente como algo dado, sino como un problema, y además, como una opción estratégica» (Ibíd, 88). Ésta es la tesis con la que pretende distanciarse de Agamben y, a la vez, poner distancias con la historia conceptual. Con la ruptura de los trascendentales y las guerras de religión en las que se ubica la génesis de la modernidad, «cayeron las defensas naturales que hasta cierto punto habían constituido el caparazón de protección simbólica de la experiencia humana», planteando la necesidad de la conservación de la vida a través de «un apartado defensivo distinto, de tipo artificial, orientado a asegurar un mundo ya constitutivamente expuesto al peligro» (Ibíd, 89). La función de mediación protectora, de envoltorio inmunitario artificial es el que cumplen las grandes categorías políticas de la modernidad, entendidas como «formas lingüísticas e institucionales adaptadas por la lógica inmunitaria para asegurar la vida contra los peligros derivados de su configuración (y conflagración) colectiva» (Ibid, 89-90).

Coincidiendo con Duso, Esposito rastrea la génesis de este dispositivo de protección negativa de la vida en el Leviatán de Hobbes. En él, se plantea con toda su radicalidad que el problema político predominante de la modernidad es la cuestión de la conservatio vitae. Hobbes parte de la constatación de que, aunque ratio y nomos naturales convergen en la conservación de la vida, entran en contradicción con la potencia expansiva de la misma: el deseo humano de poseerlo todo. De este modo, dice Esposito, «lo que pone en movimiento 
la maquinaria argumentativa hobbesiana es la circunstancia de que ni la razón ni el derecho, de por sí, alcanza ese objetivo sin un aparato más complejo que esté en condiciones de garantizarlo» (Ibid., 93). Para protegerse de la autodisolución a la que lleva esta contradicción, la vida tiene que proveerse de un mecanismo artificial que contenga y niegue su potencia expansiva, es decir, sólo negándose a sí misma a través de la mediación del artificio político, la vida puede afirmarse. Ese mecanismo artificial es, precisamente, la soberanía, primera mediación inmutaria moderna y «la más poderosa respuesta ordenadora al problema de la autoconservación» (Ibíd, 92). Coincidiendo en intenciones otra vez con Duso, Esposito está interesado en mostrar el carácter constitutivamente negativo y aporético de la inmunización en el proceso de autorización del pacto. Con el contrato que instituye la soberanía se produce un efecto de subjetivación y sometimiento: «son sujetos de ella en la medida en que la han instituido voluntariamente por medio de un libre contrato. Pero están sujeto $a$ ella porque, una vez instituida, no pueden oponérsele, por ese mismo motivo: porque se opondrían a sí mismos.» (Ibid., 96). Esposito destaca este momento para mostrar que el producto de ese sometimiento voluntario del mecanismo de la soberanía es, precisamente, la figura de la subjetividad moderna, que supone «el no ser en común de los individuos, la forma política de su disociación [...] la soberanía, en última instancia, no es sino el vacío artificial creado en torno a cada individuo, el negativo de la relación o la relación negativa entre entidades no relacionadas» (Ibid, 98).

Con lo dicho hasta aquí, se han planteado los suficientes elementos como para poder sostener que existen claras afinidades entre las dos perspectivas analizadas. De un lado, ambas sostienen que la modernidad política supone una auténtica ruptura conceptual con el horizonte de pensamiento anterior, y se erige en un dispositivo de legitimación del poder. En ambas, el concepto de soberanía asume la función de un mecanismo de ordenación de las relaciones humanas con el objetivo de neutralizar el conflicto e instaurar seguridad, mecanismo del que ambos enfoques intentan mostrar las contradicciones y aporías que les son inherentes. De otro lado, la ciencia política moderna parte del presupuesto de una conflictividad originaria que hay que neutralizar legitimando la artificialidad del poder absoluto del Estado. La capacidad neutralizadora de la modernidad y su carácter aporético se debe a la función que el concepto mismo asume; supone en ambos casos una mediación intelectual con la que se opera una abstracción de la experiencia concreta cuyo efecto performativo más evidente es la institución del sujeto como un engranaje fundamental de la máquina. En ese sentido, tanto la historia conceptual como la biopolítica ponen al descubierto que el sujeto, lejos de ser un dato inmediato, es un constructo necesario en la teoría del contrato, al incidir en la dependencia que existe, de un lado, entre subjetividad y sometimiento y, de otro lado, entre poder y derechos.

La gran diferencia que existe entre ambos planteamientos reside en sus respectivas proyecciones de este diagnóstico común, especialmente la del Esposito de Bíos. En su caso, el análisis descrito es un paso previo para ofrecer una teoría que no reduzca la gran aporía de la biopolítica moderna, la experiencia nazi, al concepto estrecho de totalitarismo, con el que a menudo se intenta dar cuenta de dos fenómenos completamente inconmensurables, como son el comunismo soviético y el nacionalsocialismo. Este interés es el que le lleva a seguir, poniendo en práctica el proceder arqueológico foucaultiano, el nomadismo conceptual del proceso de inmunización moderno en el cruce de los léxicos político y médico durante el 
siglo XIX. El proyecto espositiano propone pensar una biopolítica afirmativa que compatibilice la dimensión protectora, inmunataria de la política, evidenciada por Hobbes, con la potencia expansiva de la vida que ponen de manifiesto, entre otros, Maquiavelo o Spinoza. Más allá del intento espositiano de invertir los dispositivos tanatopolíticos nazis, esa última referencia a pensadores que se encuentran fuera del dispositivo de la modernidad es donde se puede volver a entablar un diálogo productivo entre historia conceptual y ontología de la actualidad.

\section{Pensar más allá del dispositivo de la modernidad}

Volviendo a lo que se decía un poco más arriba, ambos enfoques ponen de manifiesto que la ciencia política moderna parte, como de un presupuesto básico, del carácter originariamente conflictivo de la naturaleza humana, un conflicto dañino, que pone en peligro la vida y que hay que neutralizar con un poder capaz de aplacarlo. Analizando la modernidad como un dispositivo del orden, evidencian que esta noción de conflicto es un presupuesto necesario de su legitimación del poder, el opuesto dialéctico que pone en marcha la maquinaria. Puesta al descubierto esta lógica, ambos horizontes coinciden en reconocer que hay otras modalidades de pensar lo político que no lo reducen a lo jurídico y, por ello, capaces de proporcionar herramientas con las que pensarlo más allá de la forma-Estado. Muy brevemente, es el caso concreto de la realidad política de Europa, a la que Esposito ha dedicado el final de su última obra, Da fuori, cuyo subtítulo reza, precisamente, Una filosofía para Europa. Partiendo de su actual crisis política y económica, que «lejos de relacionarse en sus diferencias, sus partes aparecen dispersas en una multiplicidad desenraizada que ni tan siquiera tiene la fuerza constituyente del conflicto» (Esposito, 2016, 3) y que amenaza con escindirla, el autor napolitano propone volver a pensar la categoría de pueblo a la luz de la productividad del conflicto maquiaveliana, no reductible a la conceptualidad moderna. En ella se revela, al margen de las coordenadas de la soberanía, que, «antes que un conjunto indiferenciado y homogéneo ya formado, [el pueblo] está constituido por una tensión y un conflicto inextinguible» (Ibíd, 237) que ha de pensarse de forma productiva.

Muchos años antes, desde la Logica del potere (1999) Duso ya había advertido, por un lado, la discordancia constitutiva entre la Unión Europea y los conceptos políticos modernos y, por otro lado, la necesidad de «que para pensar esa nueva realidad política en construcción [...] necesitamos agenciarnos unos instrumentos conceptuales nuevos» (Duso, 2009, 327). Si se quiere entender efectivamente la Unión, se necesitan instrumentos distintos a los del ius publicum europaeum, que no la reduzcan a un mega-Estado o a una mera unión bancaria. Sólo así se la podrá concebir como «una pluralidad de sujetos que quieren confluir en una comunidad sin desaparecer como sujetos políticos, manteniendo su identidad y la posibilidad de participación política» (Ibíd., 330). Para ello, Duso avanza la posibilidad de una modalidad federalista de pensar Europa reivindicando la Política de Althusius, con la que reaparece la cuestión del gobierno -no equiparable al poder soberano y la representación- como una instancia de unificación que mantiene la politicidad de los sujetos políticos, entendidos como partes y agregaciones diferentes y diversas entre sí. 
Considero que es en esta dirección, en la remisión a modalidades y autores que se encuentran, temporal y conceptualmente, más acá o más allá de la ruptura que supone el modo moderno de entender lo político, donde debe seguir profundizándose en un diálogo fructífero entre las perspectivas abiertas por Duso y Esposito. En dicho diálogo se podrán encontrar herramientas con las que pensar, nuevamente, la comunidad, la pluralidad política y el principio de gobierno.

\section{Bibliografía}

Chignola, S. y Duso, G. (2009), Historia de los conceptos y filosofía política, Madrid, Biblioteca Nueva.

Duso, G. (ed.) (1987), Il contratto sociale nella filosofia politica moderna, Bologna, il Mulino.

Duso, G. (1999), El poder. Para una historia de la filosofía política moderna, México, Siglo XXI.

Duso, G. (1998), «Historia conceptual como filosofía política», Res Publica, n ${ }^{\circ}$, pp. 35-71.

Duso, G. (2011), «¿Qué conceptos políticos para Europa?», en: F. Oncina (ed.) (2011), Teorías y prácticas de la Historia Conceptual, Plaza y Valdés/CSIC, Madrid/México, pp. 327-348.

Duso, G. (2016), «Historia conceptual: ¿crítica o filosofía?», en F. Oncina y J.M. Romero (2016) (eds.), La historia sedimentada en los conceptos, Granda, Comares, pp. 29-48.

Esposito, R. (2006), «Storia dei concetti e ontologia dell'attualità», Filosofia Politica, XX, 1, pp. $5-9$.

Esposito, R. (2008), «La politica al presente», en Bazzicalupo, L. (2008), Impersonale. In dialogo con Roberto Esposito, Milano, Mimesis, pp. 13-37.

Esposito, R. (2011), Bíos. Biopolítica y filosofía, Buenos Aires, Amorrortu.

Esposito, R. (2015), Pensamiento viviente. Origen y actualidad de la filosofía italiana, Buenos Aires, Amorrortu.

Esposito, R. (2016a), Da fuori. Una filosofia per l'Europa, Einaudi, Torino.

Esposito, R. (2016b), «German Philosophy, French Theory, Italian Thought», Pasajes de pensamiento contemporéneo, $\mathrm{n}^{\circ} 49$, pp. 4-13.

Gentili, D. (ed.) (2007), La crisi del politico. Antologia de «Il Centauro», Guida, Napoli.

Gentili, D. (2011), Italian Theory. Dall'operaismo alla biopolitica, Bologna, il Mulino.

Sánchez Mandingorra, J. (2016), «El movimiento di pensiero de Giuseppe Duso entre historia conceptual y filosofía política», Pasajes. Revista de pensamiento contemporáneo, no 49 , pp. 50-62. 
